\title{
Complimentary Effect of L Citrulline Supplementation on Shock Wave Therapy (SWT) Induced Angiogenesis
}

\section{Short Communication}

SWT is a modern day non invasive treatment option for a wide range of condition which includes ischemic heart disease, vasculogenic erectile dysfunction, soft tissue wound, traumatized bones, kidney stones etc. It works on the basis of Angiogenesis which promotes tissue healing by the means of vascularization of the affected tissue that eventually results into tissue regeneration thus rapid healing takes place. It induces improved hemodynamic in the affected tissue which is vital for healing process.

$\mathrm{L}$ citrulline is a non protein amino acid that converts into another amino acid named $\mathrm{l}$ arginine which is the precursor of nitric oxide. Nitric oxide is essential for vasodilation as it relaxes the blood vessels, thus it improves hemodynamics which plays an important role in the process of Angiogenesis.

$\mathrm{L}$ citrulline supplementation is more effective than $\mathrm{l}$ arginine supplementation, because it promotes more bioavailability of $\mathrm{L}$ arginine than l arginine supplementation itself due to its less presystemic and systemic absorption rate in the stomach and in the liver.

The concept is all about utilizing $\mathrm{L}$ citrulline supplementation to increase the efficacy of SWT.

\section{Methods and Materials}

SWT causes micro tears in the local small blood vessels and as a response the body releases angiogenic growth factors such as eNOS, VEGF, PCNA etc. Thus induces the process of Angiogenesis. The enzymatic action of eNOS converts L arginine into nitric oxide
Short Communication

Volume 6 Issue 1 - 2017

Zaman Mirza*

Kharkiv National Medical University, Ukraine

*Corresponding author: Zaman Mirza, Kharkiv National Medical University, Prospect lenina 4, Kharkiv, Ukraine, Tel: +380934706072; Email: mirzaniaz1987@yahoo.com

Received: December 06, 2016 | Published: March 23, 2017

to induce vasodilation which promotes angiogenesis and as the process of Angiogenesis progresses, the required amount of Nitric oxide thus l arginine increases as well.

\section{Results and Discussion}

L citrulline supplementation ensures a constant bioavailability of $\mathrm{L}$ arginine which meets the increase requirement thus assisting the process of Angiogenesis induced by SWT to be continued without any disruption.

\section{Conclusion}

L citrulline supplementation can be utilized to support, accelerate and continue the process of SWT induced angiogenesis. 